Voix et Images

\title{
André Major : l'interrogation des formes
}

\section{Robert Dion et Élisabeth Nardout-Lafarge}

Volume 40, numéro 3 (120), printemps-été 2015

André Major

URI : https://id.erudit.org/iderudit/1032630ar

DOI : https://doi.org/10.7202/1032630ar

Aller au sommaire du numéro

Éditeur(s)

Université du Québec à Montréal

ISSN

0318-9201 (imprimé)

1705-933X (numérique)

Découvrir la revue

Citer ce document

Dion, R. \& Nardout-Lafarge, É. (2015). André Major : l'interrogation des formes.

Voix et Images, 40(3), 7-11. https://doi.org/10.7202/1032630ar d'utilisation que vous pouvez consulter en ligne.

https://apropos.erudit.org/fr/usagers/politique-dutilisation/ 


\title{
A NDRÉ MAJOR： L'INTERROGATION DES FORMES
}

\author{
$+++$ \\ ROBERT DION \\ Université du Québec à Montréal \\ ÉLISABETH NARDOUT-LAFARGE \\ Université de Montréal
}

C'est, comme la revue ne l'a fait que pour de rares écrivains, le deuxième dossier que Voix et Images consacre à André Major, près de trente ans après celui qu'elle lui dédiait en 1985 (vol. X, nº 3, sous la responsabilité d'André Vanasse). Deux raisons principales justifient à nos yeux cette relecture. D'une part, l'œuvre d'André Major, reconnue notamment par le prix Athanase-David en 1992, saluée par la critique comme le montre la mise à jour bibliographique que nous proposons, analysée dans des mémoires et des thèses, n'a pourtant pas, à ce jour, retenu toute l'attention critique qu'elle mérite dans les études de littérature québécoise. Longtemps cantonnée à une lecture d'abord politique, elle a sans doute pâti d'une association trop systématique à cet enjeu. D'autre part, cette œuvre s'est en bonne partie redéfinie et repositionnée aux marges de la littérature par la pratique, revendiquée comme quasi exclusive, du carnet (Le sourire d'Anton ou l'adieu au roman ${ }^{1}$ [2001], L'esprit vagabond [2007] et Prendre le large [2012], les trois livres couvrant la période de 1975 à 2000). Que cet «adieu au roman», à la fiction et à la littérature canonique n'ait pas été absolu, ce dont témoigne la publication de deux romans, La vie provisoire et À quoi ça rime?, illustre à quel point ce débat sur les genres et l'interrogation sur la fonction de la littérature dynamisent l'écriture d'André Major, qui s'en explique d'ailleurs dans l'entretien que nous publions dans ce dossier.

Cas à peu près unique dans la littérature québécoise, Major, qui a contribué à la définir et à la promouvoir, entend n'y participer qu'à partir d'un écart, d'une certaine «retraite» maintes fois figurée et thématisée dans ses écrits, et bien avant la rédaction des carnets, comme le montre le dossier. L'originalité et le paradoxe de cette position - et des textes qui l'aménagent et la défendent - nous semblent en justifier l'examen, à la fois dans les publications les plus récentes et dans l'ensemble de l'œuvre, qui gagne à être ainsi rétrospectivement réévaluée. Lue depuis les derniers tomes des Carnets, l'œuvre d'André Major impose en effet une forte cohérence, aussi bien thématique que formelle et peut-être plus encore éthique, cohérence que

1 Pour les références complètes aux œuvres de Major, le lecteur pourra consulter la bibliographie préparée par Jean-François Thériault, dans le présent dossier, p. 103-111. 
soulignent les nombreux échos qu'on trouvera entre les articles de ce dossier et l'entretien que l'écrivain nous a accordé.

Dans cet entretien, réalisé au cours de l'été 2014, Major insiste à plusieurs reprises sur les tensions qui structurent son univers et qu'il perçoit, dès «ses premières tentatives d'écriture», entre la fiction et «la prose émiettée des carnets»; entre la note prise au hasard d'une promenade et le travail de récriture et d'épure qui autorise à la donner à lire; entre la ville et les «bois montueux» du frère MarieVictorin; entre la tentation du silence et le désir de communiquer avec le lecteur. Loin de chercher à les résoudre, André Major situe dans ces tensions aussi bien ses lectures que son travail d'écrivain, qui en est indissociable. Tenant à distance les mondanités et les prétentions de la littérature au profit d'une saisie de l'élémentaire, il positionne sa pratique dans une recherche où l'authenticité commande à l'esthétique et non l'inverse. Il revendique fermement «la concision du vocabulaire, la netteté du trait et le rythme de la phrase", à l'écart des modes, sans jamais renier le réalisme. Outre cette fidélité à un souci de la forme qui vaut pour l'ensemble de l'œuvre ressort aussi fortement des propos d'André Major la place singulière qu'occupe la dimension autobiographique dans son écriture. En effet, l'expérience personnelle qui inspire la fiction ou s'analyse dans les carnets, répondant à un désir de compréhension, d'élucidation, n'a de sens que par rapport à l'expérience commune, dans le partage avec le lecteur. L'écriture de Major, privilégiant la sobriété linguistique et cherchant à s'effacer devant ce qui est "donné à voir», oppose aux ambitions de la littérature la tâche plus humble qui consiste à sauver de l'indifférence «la vie ordinaire» et l'espoir, «sinon de surmonter son désarroi, du moins [de] le partager avec ses semblables».

Les articles du dossier font écho à ces questions de multiples manières, tout particulièrement par l'attention qu'ils portent à l'interférence entre les genres, plus ou moins souterrainement présente dans l'ensemble du corpus. Ainsi, plutôt qu'un découpage de l'œuvre de Major en «périodes» qui correspondraient à des modes d'écriture distincts, ces lectures cherchent bien davantage à cerner, dès les débuts de l'œuvre et jusqu'à ses plus récents développements, une constante interrogation de la forme, au-delà des genres et souvent dans leur entrecroisement.

Dans «Le réalisme inactuel d'André Major», Michel Biron montre la dimension polémique des choix esthétiques qui président à la composition des Histoires de déserteurs, choix opérant comme autant de "déplacements» par rapport à la littérature québécoise des années 1960 et 1970; «il s'agit, écrit-il, de construire plutôt que de déconstruire, d'aller vers le lecteur plutôt que de lui tourner le dos, de revendiquer une filiation plutôt que de faire table rase et surtout de situer son œuvre dans un horizon littéraire plutôt que national ${ }^{3}$ ». Or, non sans paradoxe, la trilogie "emprunte la forme la plus romanesque qui soit, celle du roman policier, mais s'en détache comme d'un vêtement trop encombrant pour se donner la liberté d'une comédie humaine

2 Élisabeth Nardout-Lafarge, "Entretien avec André Major», dans le présent dossier, p. 27.

3 Michel Biron, «Le réalisme inactuel d'André Major», dans le présent dossier, p. 44. 
discontinue ${ }^{4}$, ce qui conduit Michel Biron à considérer, comme le constatent également les autres auteurs des textes de ce dossier, que «l'adieu au roman dont parlera Major dans ses carnets est déjà lisible dans cette œuvre de transition ${ }^{5}$ ».

André Lamontagne, dans «La médiation intertextuelle. Lecture et altérité dans La vie provisoire et À quoi ça rime?", explore des romans dont l'écriture est contemporaine de celle des carnets et en cela marquée, selon lui, par «un désenchantement narratif et un recadrage du côté de la littérature intimiste ${ }^{6} »$. Notant l'apparition dans ces deux textes d'un «nouveau type de déserteur [...]: un ermite érudit en quête de soi $^{7}$ » qui n'est pas sans évoquer l'énonciateur des carnets, Lamontagne met en perspective, d'une part, les marqueurs de l'altérité que sont l'extraterritorialité fictive (la République dominicaine dans La vie provisoire, Lisbonne dans À quoi ça rime?) et les personnages d'étrangères (Nina l'Ukrainienne, Farah l'Égyptienne, Lydia qui se sent différente en Provence de celle qu'elle est à Lisbonne), et, d'autre part, l'intertextualité «appelée à jouer un rôle central comme mode d'interprétation de la réalité et comme médiation entre moi et l'autre ${ }^{8}$ ». Dans ces "romans de la filiation", l'acte de lecture est à la fois dépaysement, comme l'exprime Antoine dans À quoi ça rime? ( «J'avais à peine achevé ma relecture de Pessoa que le besoin de lire n'importe quel roman russe me tenaillait ${ }^{9}$.»), et appel aux intercesseurs littéraires. L'article de Lamontagne relève enfin le motif de «l'ermitage» développé dans les deux romans, où il est également lié à la lecture, figurant par les livres la présence de l'altérité. Cet ermitage ouvert à l'autre, en quelque sorte paradoxalement partagé, renvoie aux propos de Major, qui revient dans l'entretien sur l'équilibre qu'il construit entre sa tentation de déserter et son besoin de dialogue.

Dans «Une forme supérieure d'intimité. Fragments de la correspondance d'André Major avec Pierre Vadeboncœur », Frédéric Rondeau aborde pour sa part un aspect de l'œuvre, l'épistolarité, négligé jusque-là par la critique malgré la publication de deux recueils de lettres ${ }^{10}$. L'article est consacré à la correspondance inédite ${ }^{11}$ avec l'essayiste Pierre Vadeboncœur, dont Michel Biron évoque ici même les pages qu'il a consacrées au personnage de Momo dans Les deux royaumes ${ }^{12}$. Frédéric Rondeau montre comment «l'activité épistolière d'André Major est pleinement cohérente avec $l^{\prime}$ ensemble de son œuvre ${ }^{13}$ » en ce qu'elle est fondée, note-t-il, sur la même nécessité

4 Ibid., p. 51.

5 Ibid.

6 André Lamontagne, «La médiation intertextuelle. Lecture et altérité dans La vie provisoire et À quoi ça rime?", dans le présent dossier, p. 53.

7 Ibid.

8 Ibid., p. 54.

9 André Major, À quoi ça rime?, Montréal, Boréal, 2013, p. 53.

10 Félix-Antoine Savard, Fraternellement... Lettres de Menaud à André Major, 1965-1971, présentées et annotées par André Major, Montréal, Leméac, coll. «Documents», 1997, 115 p.; et Jacques Ferron et André Major, «Nous ferons nos comptes plus tard». Correspondance, 1962-1983, Montréal, Lanctôt, coll. «Cahiers Jacques-Ferron", 2004, 126 p.

11 La publication de cette correspondance est toutefois prévue au Boréal à l'automne (André Major et Pierre Vadeboncœur, Nous rencontrer à mi-chemin. Correspondance, Montréal, coll. «Papiers collés», 2015).

12 Pierre Vadeboncœur, Les deux royaumes, Montréal, l'Hexagone, 1978, 239 p.

13 Frédéric Rondeau, «Une forme supérieure d'intimité. Fragments de la correspondance d'André Major avec Pierre Vadeboncœur», dans le présent dossier, p. 84. 
de «demeurer au plus près du réel, dans un mélange de simplicité, d'intimité, refusant tout hermétisme ${ }^{14} »$. Placée sous le signe d'une franchise exigeante, cette correspondance est aussi traversée par des "divergences» qui requièrent de chaque correspondant qu'il s'explique davantage; c'est le cas lorsqu'André Major reçoit en 1977 le Prix du Gouverneur général pour Les rescapés, de même qu'après la lecture, déstabilisée et déstabilisante, que Pierre Vadeboncœur fait de L'adieu au roman.

Enfin, l'article de Robert Dion et Manon Auger, «L'engagement et l'écriture (de fiction) à l'épreuve de l'autobiographique chez André Major», propose «une révision de sa trajectoire d'écrivain" en suivant «la trace autobiographique qui accompagne, en pointillé, le déploiement de la fiction ${ }^{15}$ » dans des textes moins connus, particulièrement les «Mémoires d'un jeune Canoque» publiés dans L'Action nationale en 1965 et en 1966. Observant la constance avec laquelle l'œuvre hésite et oscille entre une poétique romanesque et une poétique autobiographique, toujours habitée par «la question des rapports au collectif et à l'individuel, au social et à l'intime ${ }^{16}{ }^{\prime}$, Auger et Dion, en accord avec Robert Major évoquant le "parcours heurté d'un écrivain qui n'est fidèle qu'à lui-même et à ses aspirations profondes, sous l'écume des soubresauts ${ }^{17}$ ", concluent à une "poétique de l'ambivalence» qui trouve sa forme la plus accomplie dans les carnets. Pourtant, si Le sourire d'Anton, L'esprit vagabond et Prendre le large «viennent en quelque sorte recatégoriser après coup les confessions éparses publiées auparavant ${ }^{18}{ }^{\prime}$, le parcours est complexe, comme l'atteste le glissement, que soulignent ici les auteurs, du journal vers le carnet, alors que l'autobiographe se double de l'essayiste, voire du poète.

Attachés à des aspects différents de l'œuvre, les articles de ce dossier se rejoignent pour mettre au jour la forte cohérence qui s'en dégage et la singularise. Saisie au long d'un demi-siècle d'écriture, cette cohérence tient pour une part à la conscience de la précarité dont témoignent aussi bien la diversité des genres pratiqués et leur traitement souvent oblique que les périodes de silence ou encore les épures successives - souvent beaucoup plus conséquentes qu'il n'y paraît - auxquelles ont donné lieu les rééditions de ses livres. Tout se passe comme si l'œuvre se constituait en se remettant en jeu, cheminant, empruntant des chemins de traverse et interrogeant sa propre existence, sa validité et sa possibilité même. Le dialogue ouvert avec d'autres œuvres, cet «aréopage» mélancolique qui accompagne l'écrivain dans ses différents ermitages, lui aussi présent dès les premiers textes, alimente cette interrogation.

14 Ibid., p. 70

15 Manon Auger et Robert Dion, «L'engagement et l'écriture (de fiction) à l'épreuve de l'autobiographique chez André Major», dans le présent dossier, p. 88.

16 Ibid.

17 Robert Major, «André Major ou le métier de vivre», François Gallays, Sylvain Simard et Robert Vigneault (dir.), Le roman contemporain au Québec, Montréal, Fides, coll. «Archives des lettres canadiennes», 1992, p. 332.

18 Manon Auger et Robert Dion, «L'engagement et l'écriture (de fiction) à l'épreuve de l'autobiographique chez André Major», p. 101. 
La cohérence de l'œuvre d'André Major tient enfin à la constance d'une forme volontairement économe, contenue par la crainte de la tricherie esthétisante et la défiance à l'endroit d'un lyrisme facile, dans laquelle le travail de l'écriture doit parvenir à l'invisibilité de manière à conduire le lecteur vers la seule sensation. Dans un mouvement à bien des égards parallèle, chez Major, l'écriture de soi, qu'elle soit méditation sur sa propre vie ou rêverie sur ses lectures, n'a de sens que si elle conduit hors de soi, dans une communauté où le plus intime se révèle aussi le plus commun, le plus aisément partageable de l'expérience humaine. 